\title{
Feasibility and safety of a novel electrosurgery device as part of multi-modal bronchoscopic therapy for malignant central airway lesions
}

\author{
Abhinav Agrawal ${ }^{1}$, Udit Chaddha ${ }^{2}$, Baris Demirkol ${ }^{3}$, Septimiu Murgu ${ }^{4}$ \\ ${ }^{1}$ Interventional Pulmonology, Division of Pulmonary, Critical Care \& Sleep Medicine, Zucker School of Medicine at Hofstra/Northwell, Hempstead, \\ NY, USA; ${ }^{2}$ Interventional Pulmonology, Icahn School of Medicine at Mount Sinai, New York, NY, USA; ${ }^{3}$ Department of Pulmonary Diseases, \\ University of Health Sciences/Yedikule Chest Diseases and Thoracic Surgery Health Practice and Research Center, Istanbul, Turkey; ${ }^{4}$ Interventional \\ Pulmonology, Section of Pulmonary \& Critical Care Medicine, University of Chicago Medicine, Chicago, IL, USA \\ Correspondence to: Abhinav Agrawal, MD. Interventional Pulmonology, Division of Pulmonary, Critical Care \& Sleep Medicine, Zucker School of \\ Medicine at Hofstra/Northwell, Hempstead, NY, USA. Email: abhinav72@gmail.com.
}

Submitted Sep 29, 2020. Accepted for publication Jan 22, 2021.

doi: 10.21037/jtd-20-3001

View this article at: http://dx.doi.org/10.21037/jtd-20-3001

\section{Background}

Bronchoscopic interventions for restoring airway patency in patients with malignant central airway obstruction (CAO) have been proven to improve quality of life and lung function. Observational studies also suggest improvement in survival and successful weaning off mechanical ventilation in patients with $\mathrm{CAO}$ and respiratory failure (1-4). Patients with tracheal and mainstem bronchial obstruction and those with a high baseline dyspnea score are likely to have the greatest benefit from therapeutic bronchoscopic procedures (5). Studies show that survival in patients with malignant $\mathrm{CAO}$ that undergo successful recanalization is similar to those patients without CAO despite greater morbidity and lesser use of chemotherapy (6). Thermal ablative modalities consisting of contact electrosurgery (ES), argon plasma coagulation (APC) and laser-assisted debulking are the most commonly used modalities for immediate relief of airway obstruction. In contact ES, due to a voltage difference between the delivering probe and the target tissue, the electrons flow through the body and return to the grounding plate. Tissue resistance to flow generates heat that results in different effects depending on the temperature: coagulation $\left(>60-80^{\circ} \mathrm{C}\right)$, desiccation $\left(>100{ }^{\circ} \mathrm{C}\right)$, carbonization $\left(>200^{\circ} \mathrm{C}\right)$ and vaporization $\left(>300{ }^{\circ} \mathrm{C}\right)(7-10)$. CoreCath $2.7 \mathrm{~S}$ (Medtronic Advanced Energy LLC, Portsmouth, NH, USA) is a relatively new FDA-approved, commercially available, single-use contact
ES catheter specifically designed for airway use which enables coagulation and cut functions with an integrated suction port to evacuate carbonized tissue and smoke (11). This instrument can be used through the $2.8-\mathrm{mm}$ working channel of a bronchoscope or inserted through the rigid bronchoscope. The aim of this study is to report the feasibility, safety and short-term outcomes of applying this technology in patients who undergo interventional bronchoscopic procedures for malignant CAO.

\section{Methods and materials}

We conducted a retrospective chart review of patients undergoing therapeutic bronchoscopy for malignant CAO with the use of the CoreCath $2.7 \mathrm{~S}$ device from August 2018 to May 2019. The study was conducted in accordance with the Declaration of Helsinki (as revised in 2013). The Institutional Review Board at the University of Chicago approved the study protocol (IRB19-0833). Individual consent for this retrospective analysis was waived. The medical records were reviewed for demographic information, operative details, post-operative outcomes and complications.

\section{Procedure}

Patients underwent flexible or rigid bronchoscopy for malignant CAO. All procedures included CoreCath 
2.7S-assisted tumor debulking as part of a multimodal bronchoscopic approach. All procedures were performed either in the operating room or in the bronchoscopy suite under general anesthesia with an anesthesiologist present for anesthetic management. In our institution, all rigid bronchoscopies are performed in the operating room with the side port of rigid bronchoscope being used for ventilation. Flexible bronchoscopy was performed under general anesthesia via an endotracheal tube either in the operating room or the bronchoscopy suite, depending on availability. A multimodal therapeutic approach was used in all patients. This included a combination of mechanical debulking (via forceps or rigid bronchoscopy), neodymiumdoped yttrium aluminum garnet (ND:YAG) laser, cryotherapy, argon plasma coagulation or ES (CoreCath 2.7S). Indication for stent placement and the type of stent was at the discretion of the operator. When thermal ablative modalities were used, the $\mathrm{FiO}_{2}$ was dropped to $<40 \%$ prior to application. The $\mathrm{FiO}_{2}$ was raised back up when the desired effect was achieved or in case the patient had desaturation below $88-90 \%$. Once adequate re-oxygenation was achieved, the $\mathrm{FiO}_{2}$ was dropped again $<40 \%$ for further application of the thermal ablative modality.

The CoreCath $2.7 \mathrm{~S}$ is an ES catheter meant for single use with an integrated suction port to evacuate smoke. The device can be used via a flexible bronchoscope or through the working channel of the rigid bronchoscope. It allows ablation of soft tissues through vaporization, while also providing coagulation and hemostasis along with the ability to suction coagulated and charred tissue. A blue indicator at the distal end of the device indicates when the catheter has passed the full length of the scope and is ready for activation. The catheter is activated by pressing the CUT (Yellow) or COAG (Blue) pedals on a wireless footswitch connected to an electrosurgical generator. The reported depth of penetration is $1.65 \mathrm{~mm}$ on cut mode and $1.9 \mathrm{~mm}$ on coagulation mode. The electrode is held at the target for $2-4$ seconds for optimal effect. The power setting ranges from 0 to 40 watts on CUT and 0 to 20 watts on COAG modes, respectively. Similar to other thermal ablative modalities, high power settings may result in deeper tissue effects than lower power settings. Figure 1 demonstrates a case of multimodal bronchoscopic intervention using CoreCath 2.7S. We describe a stepwise approach to the use of this technology in Figure 2.

\section{Definitions}

Technical success of the procedure was defined as restoration of the airway lumen to $>50 \%$ of the normal caliber at the end of the procedure with patent distal airways (5). In regards to complications, we assessed the intra-operative bleeding, airway perforation and post-operative respiratory failure. Bleeding was quantified as follows: minor if it was $<20 \mathrm{~mL}$ and no hemostatic interventions were required; moderate if it was $20-50 \mathrm{~mL}$ or bleeding that required use of hemostatic therapy-laser, APC, cold saline or hemostatic agent such as SURGICEL $^{\circledR}$; severe if it was $>50 \mathrm{~mL}$ or it necessitated the use of endobronchial blockers, thoracotomy, or blood transfusions. Airway perforation was defined as either airway tear noted during bronchoscopy or evidence of pneumothorax or pneumomediastinum on postoperative imaging. Post-operative respiratory failure was based on either increased oxygen requirement post-procedure or the need for non-invasive ventilation or mechanical ventilation.

\section{Statistical analysis}

Descriptive statistics are presented using mean, median and range for the demographic and procedure outcomes variables.

\section{Results}

\section{Demographics and disease characteristics}

During the study period, 12 patients underwent 15 therapeutic bronchoscopy procedures for malignant CAO with CoreCath $2.7 \mathrm{~S}$. The baseline demographics as well as clinical characteristics of these patients are listed in Table 1. The median age was 66 (range, 54 to 84). An equal number of males and females underwent the intervention. The majority of the patients had lung cancer with adenocarcinoma being the most common subtype. One patient had endobronchial carcinoid and another had endobronchial involvement from mesothelioma. Other cancers causing endobronchial involvement included one patient with breast cancer and another with esophageal cancer. The most common presenting symptom was dyspnea with other common symptoms being cough and hemoptysis. The mean American Society of Anesthesiologist Physical Status Classification (ASA) score of patients preintervention was 3.4 with a mean Eastern Cooperative Oncology Group Performance Status (ECOG) of 2.3.

\section{Procedure details and outcomes}

Purely endoluminal exophytic CAO was found in 9 of 

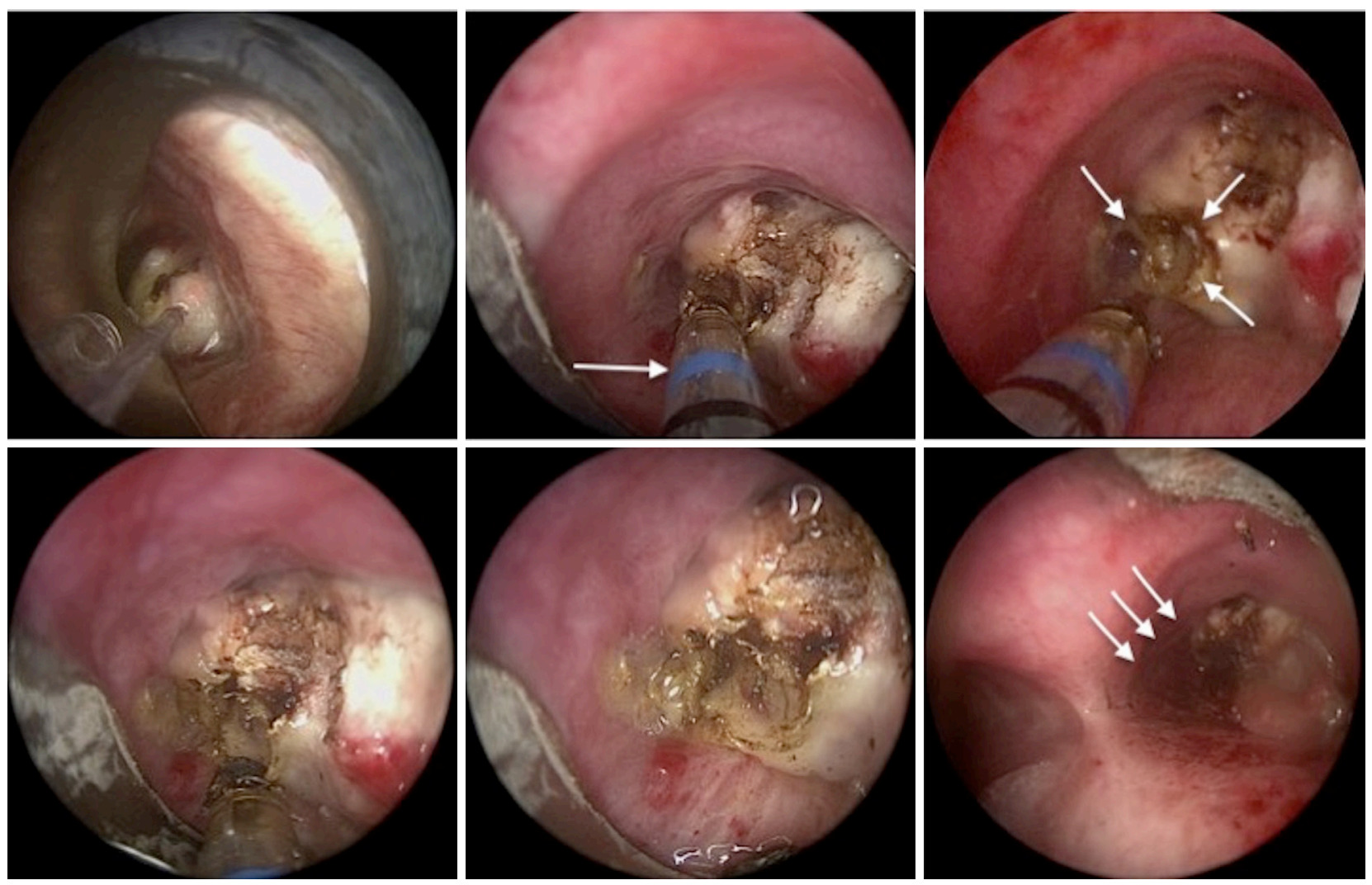

Figure 1 Multimodal bronchoscopic intervention using CoreCath 2.7S. Patient with right mainstem bronchial obstruction undergoing multimodal debulking. Top left panel: photocoagulation using low power settings with Nd:YAG laser. Note area of blanching on the lesion. Top middle panel: debulking using CoreCath 2.7S. Arrow indicates the safety mark on the catheter. Top right panel: note the area that was just cut and suctioned out by the CoreCath 2.7S (arrows). Bottom left panel: further debulking using CoreCath 2.7S while keeping the device aligned with the axis of the bronchus (co-axial). Bottom middle panel: close view of areas of tumor resected using the CoreCath 2.7S Bottom right panel: patency to the right mainstem bronchus is partially restored (arrows). Nd:YAG, neodymium-doped yttrium aluminum garnet.

the 15 procedures; in 6 procedures, there was a mixed obstruction with both exophytic component and extrinsic compression, in which case ablative therapies were used for the endoluminal component (Table 2). The majority of the lesions were located in the trachea, right mainstem or left mainstem bronchus. Ten interventions were performed using the combination of rigid and flexible bronchoscopy in the operating room, 1 procedure was performed with a flexible bronchoscope alone in the operating room using an endotracheal tube, while four procedures were performed using flexible bronchoscopy in the bronchoscopy suite using an endotracheal tube. All procedures were performed under general anesthesia with an anesthesiologist present for anesthetic management. Eight patients were on concurrent chemotherapy, immunotherapy or radiation therapy.
All procedures included CoreCath 2.7S-assisted tumor debulking as part of a multimodal bronchoscopic approach. The CoreCath 2.7S is an ES catheter meant for single use with an integrated suction port to evacuate smoke. It allows ablation of soft tissues through vaporization, while also providing coagulation and hemostasis. The catheter is activated by a by pressing the CUT (Yellow) or COAG (Blue) pedals on a wireless footswitch connected to an electrosurgical generator. The electrode is held at the target for $2-4$ seconds for optimal effect. The power setting ranges from 0 to 40 watts on CUT and 0 to 20 watts on COAG modes, respectively.

Mechanical debulking with the rigid bronchoscope or forceps was used in all 15 procedures. Nd:YAG laser was used in 8 procedures for photocoagulation prior to using CoreCath. One patient had a silicone stent placed at the 
Contact Electrosurgery (with CoreCath 2.7S) CHECKLIST

TECHNICAL SKILLS ASSESSMENT TOOL DEVELOPED BY SD MURGU, MD, FCCP, UNIVERSITY OF CHICAGO

\begin{abstract}
1. Patient safety and bronchoscopic identification of the target lesion:
- Do not use flammable anesthetics (N20, high $\mathrm{FiO}_{2}$ ) and be aware of electromagnetic interference (e.g., pacemakers, AICD)

- Assure insulation of patient (e.g., no contact with metal objects)

- Use a bronchoscope with a working channel of $2.8 \mathrm{~mm}$ or larger (flexible or rigid)

- Identify location, vertical extent and pattern (exophytic vs. infiltrative) of tumor

- Do not insert (into the bronchoscope) or withdraw the CoreCath 2.7S (from the bronchoscope) while power is being applied

- Do not touch the tip of the CoreCath 2.7S when power is being applied

- Alternate means of hemostasis should be available

2. Setting up the device system:

- Connect the CoreCath power cable into the generator receptacle

- Assure that electrical connections are tight, dry and clean

- Place device suction connector to a compatible OR suction unit (adjust suction as needed)

- Assure proper placement of the pad on patient's body and place footswitch by operator's feet

- Power on the Generator and select the desired settings: CUT 0-40 W; COAG 0-20 W; start low and change in increments of 5 W

3. Before CoreCath use:

- Assure that the $\mathrm{FiO}_{2}$ is less than 0.4 before application

- Maintain visual control of distal aspect of electrode-do not press the pedal if visibility is lost

- Keep the CoreCath co-axial (aligned with the airway to prevent perforation and bleeding)

- Do not apply CoreCath to metal clips/metal mesh/surgical sutures

- Do not apply Corecath in segmental or small lobar airways

4. Work at the target:

- Carefully insert the small metal cutting tip of the CoreCath 2.7S into the bronchoscope

- Advance electrode until the visual indicator is seen (blue band)

- Place tip of probe in front of the tumor (for exophytic dz.) or adjacently (for infiltrative dz.)

- Press the CUT (Yellow) or COAG (Blue) foot pedal for 2-4 seconds and gently advance the CoreCath into the tumor for cutting or cover the surface of tumor for coagulation effect

- Apply gentle pressure on the tissues during use and keep the electrode tip in motion while activated to avoid excessive eschar buildup

- During use, monitor the device for inadequate suction performance, which might be apparent by excessive/visible smoke or burnt odor

- Decrease or increase the power settings for the optimal cutting or coagulation effects

- Retrieve and clean the electrode with damp cloth/gauze if charred tissue is noticed at the tip
\end{abstract}

5. Final bronchoscopic inspection: complete airway inspection to rule out bleeding or residual obstructing tissues

Figure 2 A checklist approach to the use of CoreCath 2.7S.

end of the procedure.

During the study period, one attending physician performed all these procedures (SM) along with one of the two interventional pulmonology fellows (AA and UC). The degree of $\mathrm{CAO}$ was estimated by the attending physician prior to and immediately after the intervention, as per our standard practice by using the stenotic index (SI) as assessed bronchoscopically (12). The average pre-intervention SI was $85 \%$, with technical success (SI $<50 \%$ ) achieved in $13 / 15$ (87\%) of procedures. In one procedure, debulking was aborted, as there were no patent distal airways, while in another one, the procedure was aborted due to bleeding. The average immediate post-procedure SI was $34 \%$. Symptomatic improvement was noted after $87 \%$ of the procedures.
Eleven of the 12 patients (91.7\%) had a follow up surveillance bronchoscopy at a mean of 4.2 weeks and median of 4 weeks. One patient was transitioned to hospice care. The average SI on follow up bronchoscopy was $42.5 \%$. Four of the 12 patients did not require any follow up intervention after the first surveillance bronchoscopy. One patient with an indwelling stent required re-intervention at 14 days for therapeutic aspiration of secretions as part of stent maintenance but did not require any tumor debulking. The mean SI of patients not requiring repeat debulking at follow up was $14 \%$. In the other 6 patients (50\%), repeat debulking with either flexible or rigid bronchoscopy was necessary to maintain airway patency after a mean duration of 28 days. Out of the 6, rigid bronchoscopy was repeated in 1 patient 
Table 1 Demographics and clinical characteristics

\begin{tabular}{|c|c|}
\hline Patients & $\begin{array}{l}\mathrm{N}=12 \text { (12 patients } \\
\text { underwent } 15 \text { procedures) }\end{array}$ \\
\hline Age (yrs) & 66 \\
\hline \multicolumn{2}{|l|}{ Sex/gender } \\
\hline Male & 6 \\
\hline Female & 6 \\
\hline ASA score & 3.4 \\
\hline ECOG score & 2.3 \\
\hline \multicolumn{2}{|l|}{ Presenting symptoms $(n=15)$} \\
\hline Cough & 7 \\
\hline Shortness of breath & 12 \\
\hline Hemoptysis & 4 \\
\hline \multicolumn{2}{|l|}{ Comorbidities $(n=12)$} \\
\hline Chronic obstructive lung disease & 2 \\
\hline Home oxygen & 2 \\
\hline Congestive heart failure & 1 \\
\hline Interstitial lung disease & 0 \\
\hline Prior other lung disease & 0 \\
\hline \multicolumn{2}{|l|}{ Type of malignancy $(n=12)$} \\
\hline \multicolumn{2}{|l|}{ Lung cancer } \\
\hline Adenocarcinoma & 4 \\
\hline Squamous cell cancer & 3 \\
\hline Carcinoid tumor & 1 \\
\hline Non-small cell lung cancer NOS & 1 \\
\hline Mesothelioma & 1 \\
\hline \multicolumn{2}{|l|}{ Other malignancies } \\
\hline Breast cancer & 1 \\
\hline Esophageal cancer & 1 \\
\hline
\end{tabular}

ASA, American Society of Anesthesiologist Physical Status Classification; ECOG, Eastern Cooperative Oncology Group Performance Status; NOS, not otherwise specified.

after 1 week as part of the staged procedure due to extensive and prolonged debulking requirements. The average SI of patients requiring repeat intervention with debulking was $71 \%$. The tumor histology type of patients requiring repeat debulking was non-small cell lung cancer (4 patients), carcinoid tumor (1 patient) or mesothelioma (1 patient). Four
Table 2 Procedural details, lesion characteristics and outcomes ( $\mathrm{n}=15$ procedures)

\begin{tabular}{|c|c|}
\hline Procedures and outcomes variables & $\mathrm{N} /[\%]$ \\
\hline \multicolumn{2}{|l|}{ Type of CAO } \\
\hline Exophytic & 9 \\
\hline Extrinsic & 0 \\
\hline Mixed & 6 \\
\hline \multicolumn{2}{|l|}{ Location of CAO } \\
\hline Trachea & 3 \\
\hline $\begin{array}{l}\text { Right mainstem bronchus/bronchus } \\
\text { intermedius }\end{array}$ & 6 \\
\hline Left mainstem bronchus & 4 \\
\hline Right upper lobe & 2 \\
\hline Mean pre-intervention SI & $85 \%$ \\
\hline Median pre-intervention SI [IQR] & $90 \%[80-100 \%]$ \\
\hline \multicolumn{2}{|l|}{ Modality used } \\
\hline Rigid and flexible & 10 \\
\hline Flexible only & 5 \\
\hline \multicolumn{2}{|l|}{ Location } \\
\hline Operating room & 11 \\
\hline Bronchoscopy suite & 4 \\
\hline \multicolumn{2}{|l|}{ Other modalities used } \\
\hline ND:YAG laser & 8 (mean energy: $2,316 \mathrm{~J}$ ) \\
\hline Mechanical debulking & 15 \\
\hline APC & 1 \\
\hline Stent placement & 1 \\
\hline Mean post-intervention SI & $34 \%$ \\
\hline Median post-intervention SI [IQR] & $30 \%[10-50]$ \\
\hline $\begin{array}{l}\text { Technical success (defined as lumen } \\
>50 \% \text { per acquire) }\end{array}$ & $(13 / 15)^{\#}$ \\
\hline Symptomatic improvement & $13 / 15$ procedures \\
\hline $\begin{array}{l}\text { Mean SI after follow up } \\
\text { bronchoscopy/intervention }\end{array}$ & $42.5 \%$ \\
\hline \multicolumn{2}{|l|}{ Duration of follow up } \\
\hline Mean & 4.2 weeks \\
\hline Median & 4 weeks \\
\hline $\begin{array}{l}\text { Patients requiring re-intervention } \\
\text { with debulking for malignant } \mathrm{CAO}\end{array}$ & $6 / 12(50 \%)$ \\
\hline
\end{tabular}

Table 2 (continued) 
Table 2 (continued)

\begin{tabular}{lc}
\hline Procedures and outcomes variables & $\mathrm{N} /[\%]$ \\
\hline $\begin{array}{l}\text { Mean time to re-intervention for } \\
\text { debulking for recurrence of malignant }\end{array}$ & 28 days \\
CAO & \\
Average SI of patients requiring a & $71 \%$ \\
repeat debulking $(6 / 12)^{\star}$ & $14 \%$ \\
Average SI of patients not requiring \\
repeat debulking $(5 / 12)^{*}$ \\
\hline , one procedure was stopped, as there were no distal \\
airways noted and another due to bleeding; *, one patient was \\
transitioned to hospice and had no follow up bronchoscopy. \\
CAO, central airway obstruction; SI, stenotic index; Nd:YAG, \\
neodymium-doped yttrium aluminum garnet; APC, argon \\
plasma coagulation.
\end{tabular}

Table 3 Complications

\begin{tabular}{lc}
\hline Type of complication & $\mathrm{N}[\%]$ \\
\hline Bleeding & $11[73]$ \\
No bleeding & $1[7]$ \\
Mild bleeding & $1[7]$ \\
Moderate bleeding & $2[13]$ \\
Severe bleeding & 0 \\
Airway injury/perforation & 0 \\
Post-operative respiratory failure & 0 \\
Immediate procedure related mortality & \\
\hline
\end{tabular}

of the 6 patients were on concurrent chemotherapy, 1 patient received just radiation and 1 patient was not considered a candidate for any further systemic therapy.

\section{Complications}

Peri-procedural complications are summarized in Table 3. No bleeding was documented in $11 / 15$ procedures (73\%). Severe bleeding, as defined in our study, was noted in 2 procedures (13\%). Bleeding volume in both these procedures was estimated at $>50 \mathrm{~mL}$ and it was controlled endoscopically and did not require the use of endobronchial blockers, transfusions, thoracotomy or admission to the intensive care unit. One of these patients had endobronchial carcinoid (purely endoluminal disease) and the other had squamous cell carcinoma of the lung (mixed CAO). None of the procedures were complicated by peri or post-operative respiratory failure, airway perforation or mortality.

\section{Discussion}

We present one of the first human studies describing the initial experience with a new electrosurgical device for malignant CAO. We believe the study adds to the body of literature that thermal ablative interventions relieve airway obstruction and symptoms, thus allowing initiation or continuation of systemic anti-cancer therapy. This is relevant as the prevalence of $\mathrm{CAO}$ in lung cancer patients is $13 \%$ (13). Many patients have diagnostic delays and often present when are very symptomatic and require hospitalization (2). Patients are referred for bronchoscopic interventions when they have dyspnea affecting performance status or are unable to undergo chemotherapy or radiation, or when they have post obstructive pneumonia, hemoptysis or even respiratory failure. Therapeutic bronchoscopy is usually offered for improving performance status and is seen as a bridge therapy to allow administration of chemotherapy and radiotherapy $(6,14)$. In these regards, in our case series, $8 / 12$ patients $(66 \%)$ were on concurrent systemic therapy (chemotherapy, immunotherapy and/or radiation). We believe that the goal of interventional bronchoscopy is to restore airway patency safely and ideally to obtain a durable effect. A multimodal bronchoscopic approach is usually adopted by many interventionalists with the preference of therapy based on available resources and local expertise $(15,16)$. This may include other thermal therapies (contact ES, APC, laser), cryotherapy, photodynamic therapy and stenting. Case series of contact ES have been published over the years, with the majority showing improvement in the SI (85-95\%) and symptoms (47-71\%) (17-21).

In our case series using CoreCath $2.7 \mathrm{~S}$, technical success was noted in $87 \%$ of the procedures with the post-procedure SI showing significant improvement, from severe (mean SI: $85 \%$ ) to mild obstruction (mean SI: 34\%) immediately postprocedure. All of the patients in whom technical success was achieved showed symptomatic improvement. The mean duration of a follow up surveillance bronchoscopy was 4.2 weeks (median: 4 weeks). Of note, follow up bronchoscopy is per our standard operating practice. The average SI during follow up was $42.5 \%$ indicating a durable response after these interventions. In our series, $50 \%$ of the patients required a repeat procedure, with mean time to reintervention of 28 days, highlighting the relevance of follow up bronchoscopy in these patients. Re-intervention with 
debulking was pursued in patients with a SI $>50 \%$ (mean SI: $71 \%$ ), while no debulking was required in patients with SI $<50 \%$ (mean SI: $14 \%$ ). All patients who did not require debulking were receiving concomitant chemotherapy, immunotherapy or radiation therapy signifying the importance of bronchoscopy as part of a multi-modality approach to treating patients with malignant CAO.

The most concerning complication of bronchoscopic debulking is airway bleeding with its consequences on oxygenation and hemodynamics. In a previous study using conventional contact ES, the authors reported mild bleeding (controlled with ES and instillation of 2\% lidocaine with epinephrine) in $38 \%$ of the cases and moderate bleeding (precluding further tumor debulking) in $12 \%$ of the cases (20). In another study, no major bleeding was noted except in 1 case of a patient with tracheal metastasis from adenoid cystic carcinoma of the lung (21). In our study, severe bleeding was noted in 2 procedures (13\%) despite prior photocoagulation with ND:YAG laser at low power density (case 1: 2,675 Joules and case 2: 3,950 Joules). Notably, the severe bleeding rate in our study is based on the volume criterion only as it was controlled with endoscopic techniques not requiring blockers, transfusions or thoracotomy and was not associated with peri-procedure morbidity or mortality. Other reported complications from ES include airway perforation and fires $(22,23)$. We had no such complications, post-operative respiratory failure or procedure-related mortality. The lack of perforation may be technique-related (always keeping the catheter co-axial during the debulking-Figure 1) or may be explained by the average depth of penetration of 0.9 and $1.1 \mathrm{~mm}$ on the CUT and COAG modes, respectively.

Our small retrospective case series suggests that CoreCath $2.7 \mathrm{~S}$ is a safe and feasible adjuvant tool for treating patients with malignant $\mathrm{CAO}$, with a bleeding rate comparable to those reported in prior studies of contact ES. Although this device may play a synergistic role in debulking malignant $\mathrm{CAO}$, it has its limitations, which need to be acknowledged. Based on our experience to date, this device is relatively stiff and can limit access to acutely angled airways (especially the right upper lobe and left upper division bronchi). While the dual capability of ES and suctioning is helpful, the suction channel becomes frequently clogged and require cleaning with wet gauze, potentially adding time to the procedure. This is a single use, disposable device and thus the costeffectiveness needs to be considered and compared to reusable devices such as the cryotherapy probe or tools used for mechanical debulking.
We believe that a future study should evaluate the adjuvant role of CoreCath $2.7 \mathrm{~S}$ in regards to safety profile, recanalization rate, time to repeat intervention and duration of procedure, ideally in a prospective comparative trial. A recent study suggests that the only modifiable risk factor associated with mortality among patients undergoing rigid bronchoscopy is the duration of anesthesia (24). This is usually due to the need of continuously adjusting the $\mathrm{FiO}_{2}$ for thermal energy application while trying to avoid severe hypoxemia. It is possible that CoreCath $2.7 \mathrm{~S}$ may reduce the procedure duration given its coagulation, cutting and suction properties, but this hypothesis needs further study.

Our study has several limitations. This is a retrospective small case series that was performed at a single center with one operating team performing all interventions. In this retrospective study, the assessment of symptomatic improvement was subjective and we did not use validated dyspnea assessment tools. Many patients had a combination of symptoms and showed improvement after $87 \%$ of the procedures based on evaluation during the routine follow up visits. We included all patients with malignant $\mathrm{CAO}$ undergoing treatment with CoreCath $2.7 \mathrm{~S}$ and reported all additional bronchoscopic modalities. The use of several techniques and concurrent systemic treatment can be confounders for assessing true efficacy related to the use of this particular device. We believe, however, that the multi-modal approach reflects the real-world practice in treating malignant CAO. Thus, to make the results more generalizable, this tool needs to be studied by means of a multi-center prospective randomized controlled trial. It should be studied either as an adjuvant modality to the standard of practice or in a direct comparative trial with another immediate-relief bronchoscopic ablative therapy.

\section{Conclusions}

CoreCath $2.7 \mathrm{~S}$ was found to be a safe and feasible adjuvant tool for restoring airway patency in patients with malignant CAO. Comparative trials are necessary for defining its costeffectiveness.

\section{Acknowledgments}

Funding: None.

\section{Footnote}

Data Sharing Statement: Available at http://dx.doi. 
org/10.21037/jtd-20-3001

Conflicts of Interest: All authors have completed the ICMJE uniform disclosure form (available at http://dx.doi. org/10.21037/jtd-20-3001). SM serves as an educational consultant for Olympus, Boston Scientific, Pinnacle Biologics, Cook. ERBE and Johnson \& Johnson. The other authors have no conflicts of interest to declare.

Ethical Statement: The authors are accountable for all aspects of the work in ensuring that questions related to the accuracy or integrity of any part of the work are appropriately investigated and resolved. The study was conducted in accordance with the Declaration of Helsinki (as revised in 2013). The Institutional Review Board at the University of Chicago approved the study protocol (IRB190833). Individual consent for this retrospective analysis was waived.

Open Access Statement: This is an Open Access article distributed in accordance with the Creative Commons Attribution-NonCommercial-NoDerivs 4.0 International License (CC BY-NC-ND 4.0), which permits the noncommercial replication and distribution of the article with the strict proviso that no changes or edits are made and the original work is properly cited (including links to both the formal publication through the relevant DOI and the license). See: https://creativecommons.org/licenses/by-nc-nd/4.0/.

\section{References}

1. Mahmood K, Wahidi MM, Thomas S, et al. Therapeutic bronchoscopy improves spirometry, quality of life, and survival in central airway obstruction. Respiration 2015;89:404-13.

2. Murgu S, Langer S, Colt H. Bronchoscopic intervention obviates the need for continued mechanical ventilation in patients with airway obstruction and respiratory failure from inoperable non-small-cell lung cancer. Respiration 2012;84:55-61.

3. Stanopoulos IT, Beamis JF Jr, Martinez FJ, et al. Laser bronchoscopy in respiratory failure from malignant airway obstruction. Crit Care Med 1993;21:386-91.

4. Colt HG, Harrell JH. Therapeutic rigid bronchoscopy allows level of care changes in patients with acute respiratory failure from central airways obstruction. Chest 1997;112:202-6.

5. Ost DE, Ernst A, Grosu HB, et al. Therapeutic bronchoscopy for malignant central airway obstruction: success rates and impact on dyspnea and quality of life. Chest 2015;147:1282-98.

6. Verma A, Goh SK, Tai DYH, et al. Outcome of advanced lung cancer with central airway obstruction versus without central airway obstruction. ERJ Open Res 2018;4:00173-2017.

7. Chaddha U, Hogarth DK, Murgu S. Bronchoscopic ablative therapies for malignant central airway obstruction and peripheral lung tumors. Ann Am Thorac Soc 2019;16:1220-9.

8. Sachdeva A, Pickering EM, Lee HJ. From electrocautery, balloon dilatation, neodymium-doped:yttrium-aluminumgarnet (Nd:YAG) laser to argon plasma coagulation and cryotherapy. J Thorac Dis 2015;7:S363-79.

9. Mahmood K, Wahidi MM. Ablative therapies for central airway obstruction. Semin Respir Crit Care Med 2014;35:681-92.

10. Barlow DE. Endoscopic applications of electrosurgery: a review of basic principles. Gastrointest Endosc 1982;28:73-6.

11. Mahajan AK, Herdina KA, Howk KA, et al. Performance of a Novel Electrosurgical Device for Cutting and Coagulation of Central Airway Obstructions. In: D61. Imaging, interventions, and interventional pulmonology: the three "I's" of thoracic oncology. American Thoracic Society, 2018:abstr A7329.

12. Murgu S, Colt HG. Morphometric bronchoscopy in adults with central airway obstruction: case illustrations and review of the literature. Laryngoscope 2009;119:1318-24.

13. Daneshvar C, Falconer WE, Ahmed M, et al. Prevalence and outcome of central airway obstruction in patients with lung cancer. BMJ Open Respir Res 2019;6:e000429.

14. Chhajed PN, Baty F, Pless M, et al. Outcome of treated advanced non-small cell lung cancer with and without central airway obstruction. Chest 2006;130:1803-7.

15. Giovacchini CX, Kessler ER, Merrick CM, et al. Clinical and radiographic predictors of successful therapeutic bronchoscopy for the relief of malignant central airway obstruction. BMC Pulm Med 2019;19:219.

16. Wahidi MM, Herth FJF, Chen A, et al. State of the art: interventional pulmonology. Chest 2020;157:724-36.

17. Sutedja G, van Kralingen K, Schramel FM, et al. Fibreoptic bronchoscopic electrosurgery under local anaesthesia for rapid palliation in patients with central airway malignancies: a preliminary report. Thorax 1994;49:1243-6.

18. Coulter TD, Mehta AC. The heat is on: impact of endobronchial electrosurgery on the need for Nd-YAG laser photoresection. Chest 2000;118:516-21. 
19. Wahidi MM, Unroe MA, Adlakha N, et al. The use of electrocautery as the primary ablation modality for malignant and benign airway obstruction. J Thorac Oncol 2011;6:1516-20.

20. De la Cruz LI, Pereira A, Krieger BP. Use of endobronchial electrocautery for the palliation of airway obstruction due to metastases from nonpulmonary malignancies. J Bronchology Interv Pulmonol 2006;13:124-7.

21. Horinouchi H, Miyazawa T, Takada K, et al. Safety study of endobronchial electrosurgery for tracheobronchial lesions: multicenter prospective study. J Bronchology

Cite this article as: Agrawal A, Chaddha U, Demirkol B, Murgu S. Feasibility and safety of a novel electrosurgery device as part of multi-modal bronchoscopic therapy for malignant central airway lesions. J Thorac Dis 2021;13(5):3151-3159. doi: $10.21037 /$ jtd-20-3001
Interv Pulmonol 2008;15:228-32.

22. Lee P, Kupeli E, Mehta AC. Therapeutic bronchoscopy in lung cancer. Laser therapy, electrocautery, brachytherapy, stents, and photodynamic therapy. Clin Chest Med 2002;23:241-56.

23. Sheski FD, Mathur PN. Endobronchial electrosurgery: argon plasma coagulation and electrocautery. Semin Respir Crit Care Med 2004;25:367-74.

24. Murgu S, Laxmanan B, Stoy S, et al. Evaluation of safety and short-term outcomes of therapeutic rigid bronchoscopy using total intravenous anesthesia and spontaneous assisted ventilation. Respiration 2020;99:239-47. 